\title{
Effect of Microstructures and Material Compositions on Blister Formation
}

\author{
Yunan Prawoto Jern Phye Tan, Choon Yong Loh
}

Faculty of Mechanical Engineering, University Technology Malaysia, Johor, Malaysia.

Email: yunan.prawoto@gmail.com

Received May 10 $0^{\text {th }}, 2010$; revised June $13^{\text {th }}, 2010$; accepted June $25^{\text {th }}, 2010$.

\begin{abstract}
One of the early failures of coating is blister. Here two different coatings were applied on various materials and microstructures. The formation of the blister, and its microstructural and material dependencies were evaluated. It is concluded that general organic coating forms more severe blisters than that of the metallic effect coating. General organic coating protects the samples by insulation film while metallic coating protects by acting as galvanic protection. Therefore, the failure modes are also different, namely blister and filiform corrosion. The dependencies on the microstructures and on material types also follow the insulation film concept and galvanic protection concept.
\end{abstract}

Keywords: General Organic Coating, Metallic Effect Coating, Corrosion, Blister

\section{Introduction}

Organic coating is among the most widely used surface engineering modifications. This corrosion protection method allows one to choose the type of the coating according to the application. Manufacturers design substrates with the desired physical and mechanical properties. The users can choose and utilize a coating that is resistant to the environment to which the part is to be exposed according to their predictions.

Besides engineering application, coating is also widely used in non-engineering applications. Due to its wide application and availability, their usage is often erroneous; many practitioners and engineers are not careful enough to consider the composition of the base materials to be painted let alone to consider the microstructures.

This research aims to clarify the first step of the corrosion failure mode in general organic coating, which is blistering. General organic coating was applied on both ferrous and non-ferrous materials, in different microstructures. The specimens were subsequently tested using compound corrosion test mode. The formation of the blister, and its microstructural and material dependency were then evaluated.

\subsection{Coating Protection}

Coating is a method of corrosion protection by application of paint films to a metal in order to protect or insulate the metal from corrosive environment, water and oxy- gen. Coating also can be adjusted by using less noble metal so that the less noble coating such as zinc coating corrodes instead of the metal itself. Thus, corrosion can be prevented.

Organic coating is a paint containing organic elements such as plastic and rubber. The main purpose of this coating is to isolate the metal from corrosive environment such as seawater, or daily moist environment. An example of organic coating is sheet linings, which use vinyl, organisols, or plastisols. The most common form of organic coating is in the form of liquid-applied paint. This kind of paint can be applied easily to a surface by using brush, roller, or spray. This paint consists of four basic components: resin, solvent, pigments, and miscellaneous compounds. Resin is also known as binder which is the most influential in determination of resistance. Solvent can be water or organic acrylic. Pigments are added as rust inhibition, to decrease permeability, and provide color or increase the ultraviolet and weathering resistances. Normally, pigments used include zinc phosphate, zinc molybdate, zinc phosphorus silicate, zinc chromate and strontium chromate [1]. Some organic coatings use metal flake to achieve their metallic appearance. It ranges from aluminum to chromium flake. This type of coating is called metallic effect coating [2].

\subsection{Blister in Organic Coating}

In general, there are six types of corrosion related to general organic coatings. Those are: blistering, early rusting, 
flash rusting, anodic undermining, filiform corrosion, and cathodic delamination. These are in addition to other deteriorations such as loss of adhesion. Blistering is considered as the first failure mode of corrosion in coated materials.

It is also widely known that alternating between dry and wet accelerates the formation of the blister. Cycling of the dry and wet can be perfectly achieved by compound corrosion test (CCT) test that combines drying and immersions. This cycle provides more internal stress that leads to creating easy formation of blister [3].

\section{Experimental Method}

In this research, both ferrous and non-ferrous materials were used. For ferrous material, steel was selected. The variation of the heat treatment was also used as a parameter. For the non-ferrous materials, copper and aluminum-based alloys were used.

\subsection{Adhesion Test}

Adhesion test is an important test for any paint film. Poor adhesion means that the paint can be comparatively removed readily from the substrate; this is generally considered to indicate the existence of a potential problem. In this research, adhesion test was performed using cross cut method according to ASTM D 3359-05 [4]. A series of 11 parallel cuts each $1 \mathrm{~mm}$ apart, was made through the coated film to the specimen substrate of a specimen by using cutter knife or razor blade. A new cutter knife was used for next specimen in order to maintain the sharpness of the knife. Peel a piece of adhesive tape on to the cross cut area by pressing with uniform force. This can prevent bubbles from the entire area of cross cut. Subsequently, adhesive tape is then peeled.

\subsection{Corrosion Test with Scribe}

Before testing, X-cut on the coating was made with the blade of cutter knife vertically to the test piece coated surface. The length is $40 \mathrm{~mm}$ long cut in the shape of " $\mathrm{X}$ ", with the intersection forming an angle of $30^{\circ}$. The samples were then tested using compound corrosion testing mode. This consists of 15 minutes immersion in $5 \% \mathrm{NaCl}$ and $11.5 \mathrm{hrs}$ dry off periods. Total test was 60 cycles. After the testing, rinse the coating surface with clean running water, and the samples were evaluated.

\section{Results and Discussions}

\subsection{Adhesion Test}

After the cross-hatches test, it can be clearly seen that the quality of general organic coating is very good compared to that of metallic effect coating. However, as they appeared in Figure 1, the general organic coating performs better than metallic effect coating. The paint cracks when the knife blade is applied on the coating. Therefore, the adhesive quality is weak while applying the adhesive tape (see Figure 2). The destruction of metallic coating when it is applied to non-ferrous materials is more severe. This is due to the base material hardness and not due to the microstructure or materials compositions.

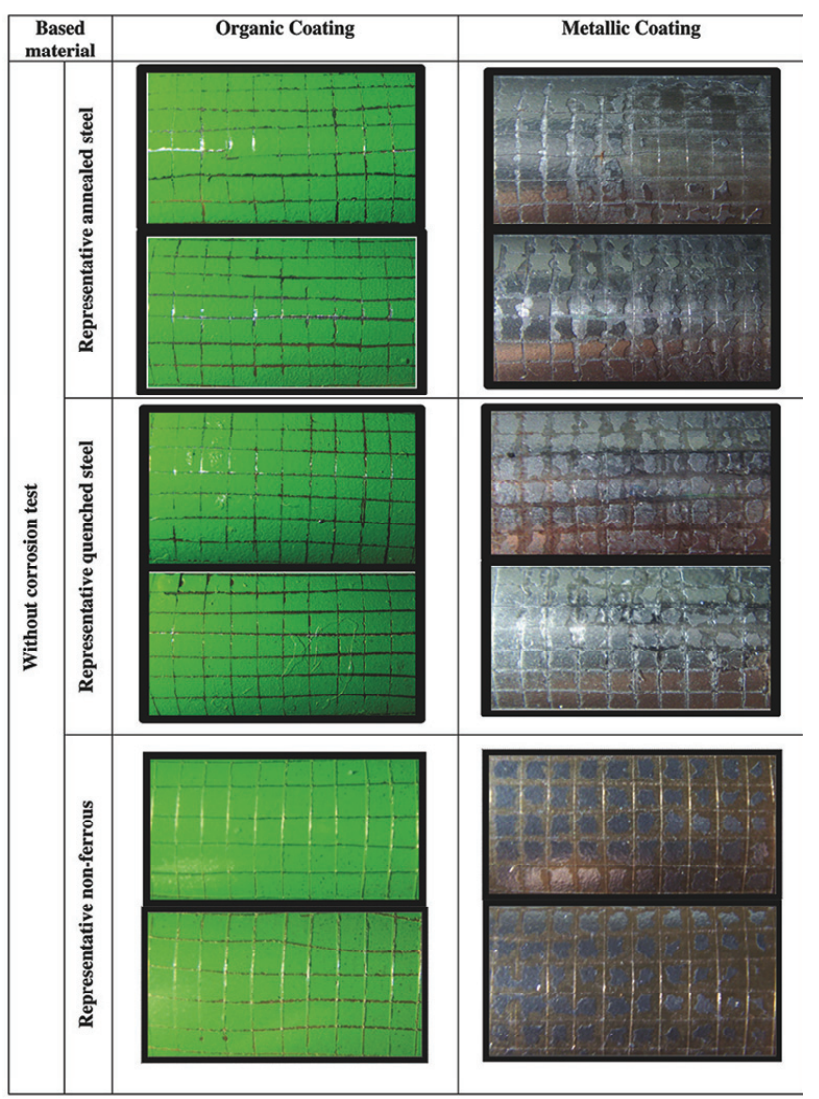

Figure 1. Appearance of the adhesion test results

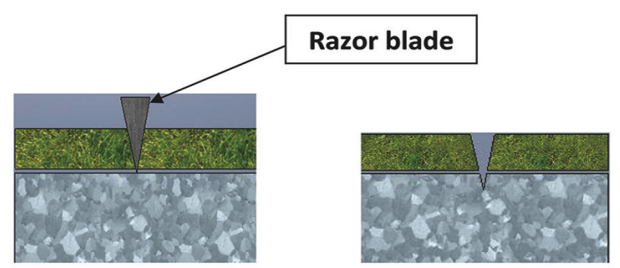

(a)

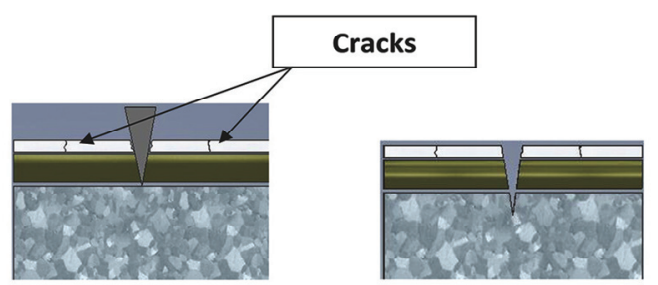

(b)

Figure 2. The effect of cutting on (a) General organic coating (b) Metallic effect coating 


\subsection{Blistering Test Results}

Figures 3(a) and 3(b) show the conditions of the blistering for ferrous materials after 60 cycles. The blister for quenched materials shows that high carbon produces fewer

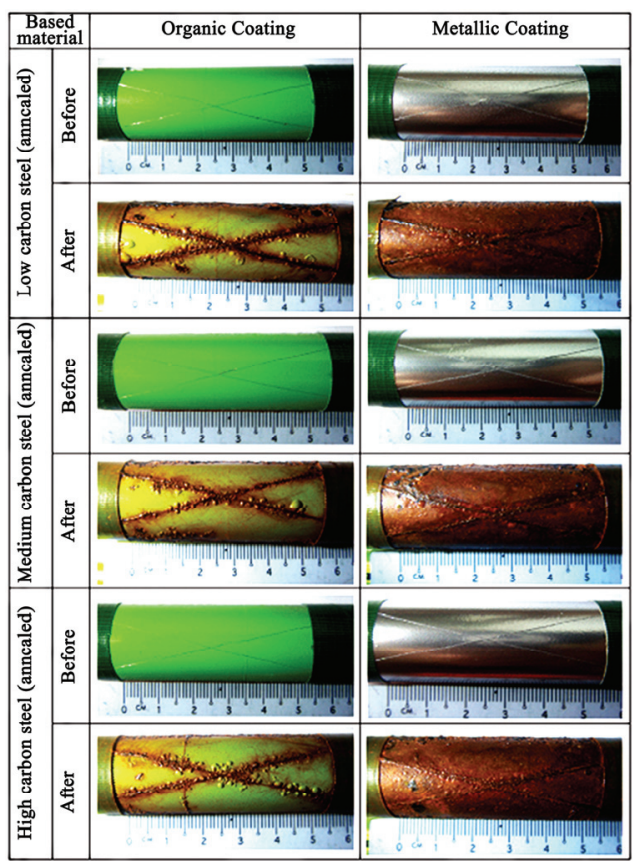

(a)

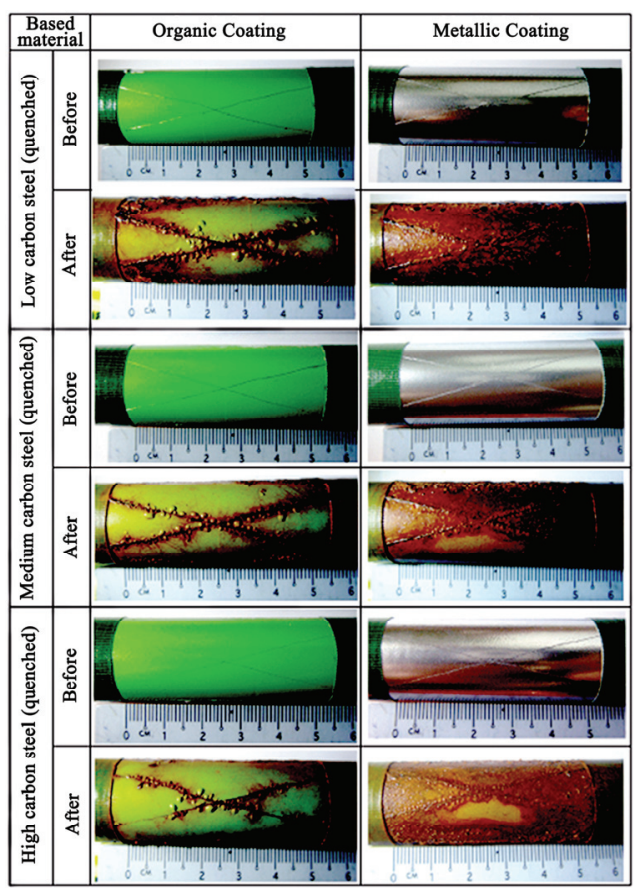

(b)

Figure 3. (a) Appearance of the annealed samples before and after 60 cycles of CCT; (b) Appearance of the quenched samples before and after 60 cycles of $\mathrm{CCT}$ blisters. This phenomenon was not observed for the annealed samples. Materials dependency on the blistering can be seen in Figure 4. It shows that aluminum produces fewer blisters than that of brass. Furthermore, with copper as the base material, blisters were found independent of the scribes.

Figure 5 shows the cross section of the blistering near and originated from the scribes. It is clear that majority of the blister is formed by corrosion product originated

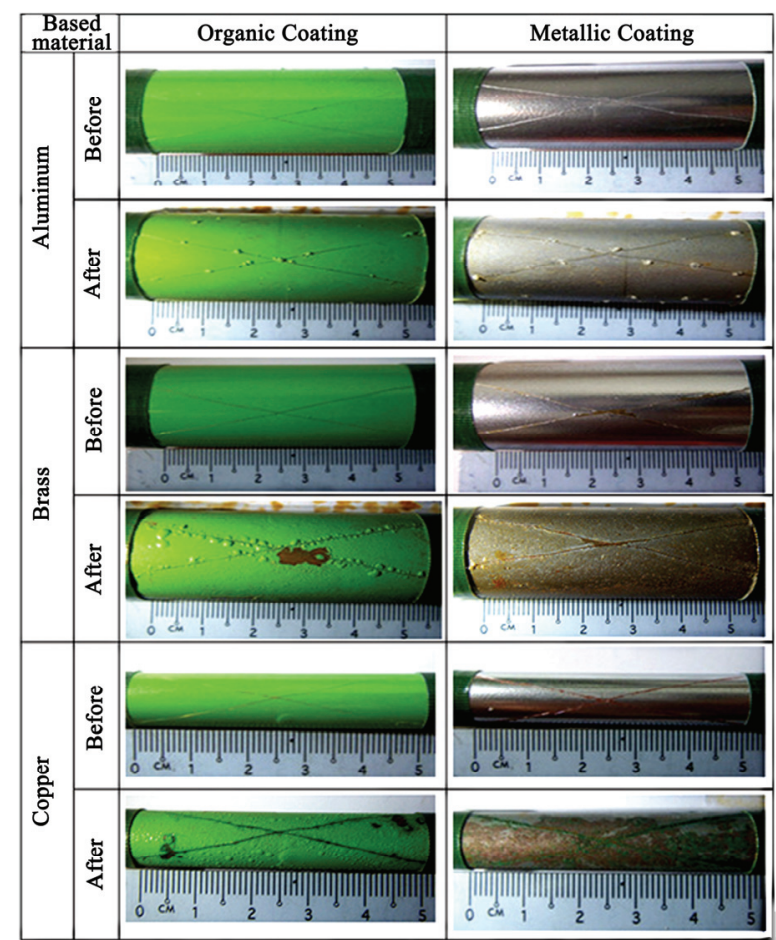

Figure 4. Appearance of the non-ferrous samples before and after 60 cycles of CCT

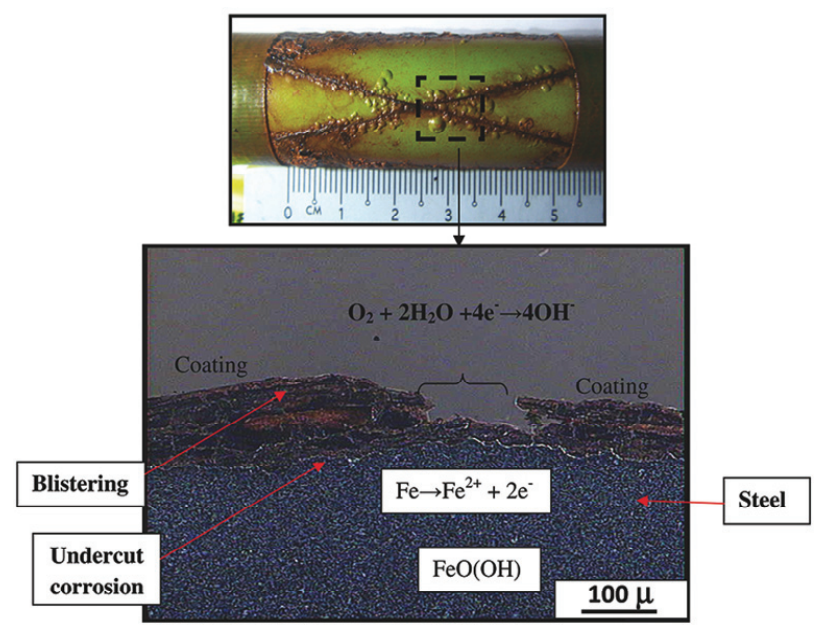

Figure 5. Cross section of scribed area in micrograph shows undercut corrosion 
from the scribes. Some blisters occur independent of the scribes. Among materials tested, copper produces most blisters that are independent of the scribe. To explore further on the mechanism, more cross sections were evaluated. Figure 6 shows the summary of the results. The figure shows that moisture can penetrate inside the coating without passing the scribe. Once the moisture and the chloride penetrate, they create corrosion that has a larger volume than the crystalline one. This large volume is the one that push the coating outside causing the blister to occur.

Figure 7 shows that regardless of the coating, the mechanism of the corrosion remains the same. Microscopi-

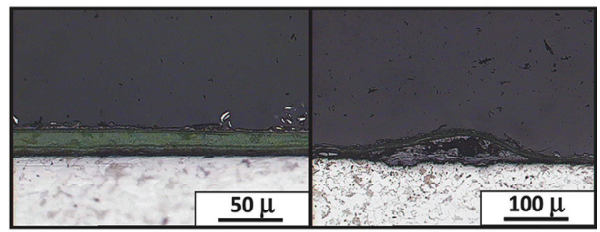

(a)

(b)

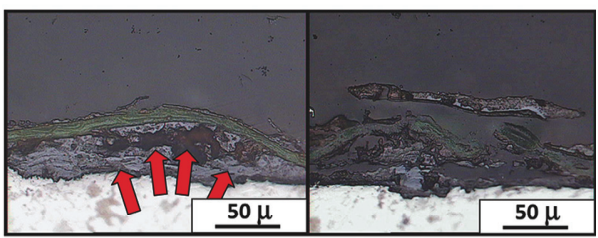

(c)

(d)

Figure 6. Blistering. (a) Normal coating; (b) Blistering formed; (c) Pressure exert
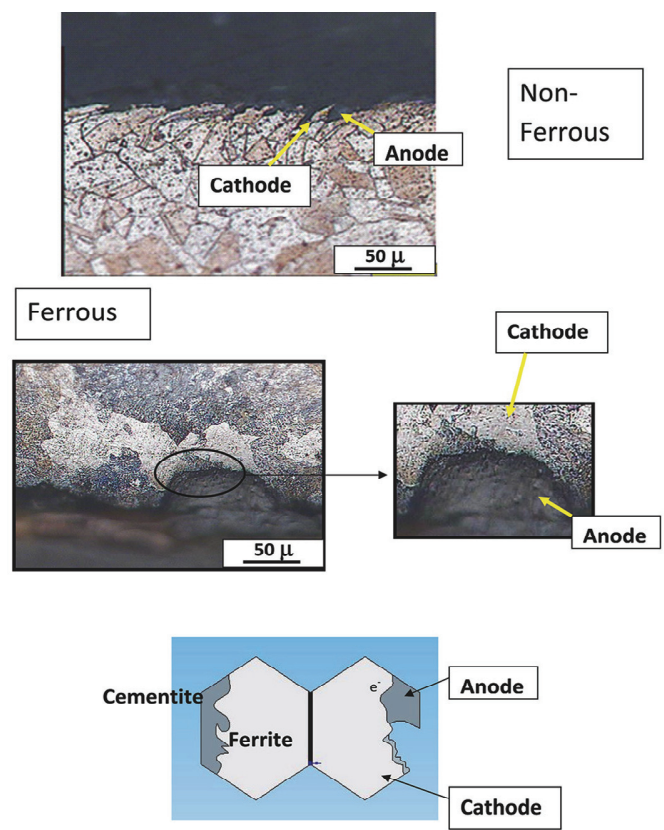

Figure 7. Corrosion mechanism in two different phases underneath the coating

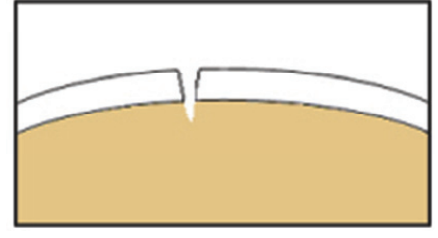

(a)

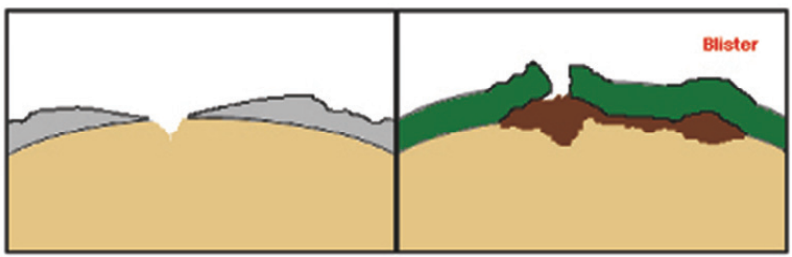

(b)

(c)

Figure 8. (a) Coating before corrosion; (b) Metallic coating after corrosion (sacrificial); (c) Organic coating after corrosion

cally, the microstructure dependency is driven by the galvanic corrosion, where cementite phase corrodes more than the ferrite phase, as they work as anode and cathode.

For the metallic effect coating, blistering was not observed. This is because the metallic flake works like sacrificing anode. Therefore, the failure mode is filiform corrosion (Figures 3(a), 3(b) and 4). Graphical explanation on the difference between metallic effect coating and general organic coating is shown in Figure 8.

\section{Conclusions}

Two representative coatings, namely organic and metallic effect coatings, were evaluated. For all based materials used, the general organic coating formed more severe blister than metallic effect coating. General organic coating protects the samples by protecting film while metallic coating provides galvanic protection. As a result, the failure mode is also different. The former forms blister while the latter forms filiform corrosion. Consequently, the dependencies on the microstructures and on material compositions are different. General organic coating follows the protecting film concept, while the metallic effect coating follows the concept of galvanic cell.

\section{REFERENCES}

[1] J. R. Davis, "Surface Engineering for Corrosion and Wear Resistance," American Society for Materials International, Materials Park, 2001.

[2] K. Stevens, "Surface Engineering to Combat Wear and Corrosion: A Design Guide," The Institute of Materials, London, 1997.

[3] Z. W. Wicks, "Organic Coatings: Science and Technology," Wiley-Interscience, Haboken, 2007.

[4] “ASTM Standard: D3359-07," ASTM Annual Book of Standards, ASTM Publisher, West Conshohocken, 2007. 